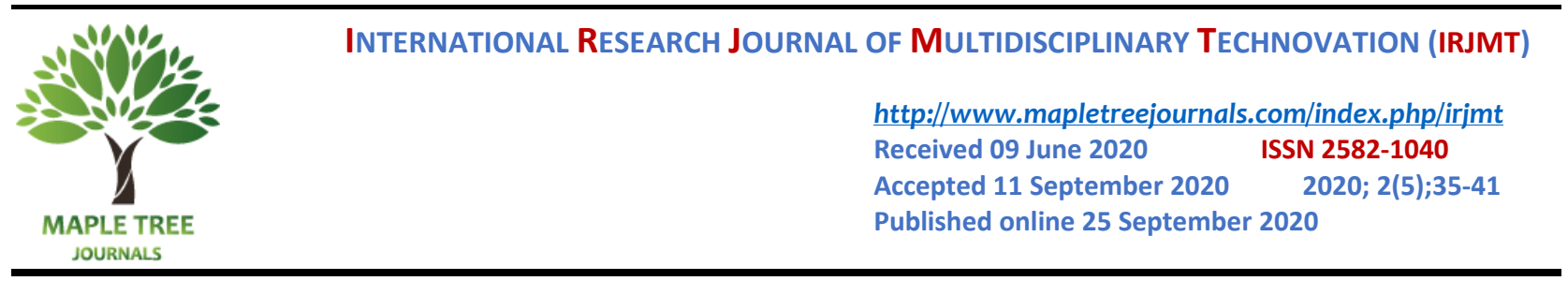

\title{
IoT Based Cloud Agent System for Adult Health Care Monitoring
}

\author{
V. Prasanna kumari ${ }^{1, *}$, C. Amutha ${ }^{1}$ \\ ${ }^{1}$ Department of Electrical and Electronics Engineering, Rajalakshmi Engineering College(Autonomous), Thandalam, \\ Chennai, Tamil Nadu, India. \\ *Corresponding author E-Mail ID: silviaprasannakumari1995@gmail.com \\ DOI: https://doi.org/10.34256/irjmt2056
}

\begin{abstract}
A continuous health monitoring system for patients with long term sickness and older age people in bed rest in-home or hospital. The system is developed to provide a solution for the problem by continuously monitor the patient's health using wireless sensor networks (WSN) and Machine learning. The system provides visual monitoring service through live video. The vital signs of the patient can be monitored such as temperature, humidity, pulse, breathing rate, etc. and provides the live monitoring using an MQTT mobile application and losant dashboard cloud server. From the collected data by using a machine learning algorithm like a random forest we can able to target the risk causing factors and rescues the patient immediately.
\end{abstract}

Keywords: Temperature sensor, pulse sensor, tilt sensor, sound sensor, MQTT protocol, Nodemcu, IOT.

\section{Introduction}

The proposed system monitors the health parameters of the patient with long term sickness and older age people who are bed return. The developed system provides a solution for those patients by continuously monitors the body's basic functions using a wireless sensor network and machine learning algorithm. The monitor of basic vital functions such as temperature, humidity, pulse, respiration, etc. and provide live monitoring using the android application and losant dashboard cloud server. From the collected sensor data, machine learning algorithm like the random forest is implemented. The collected data is then sent to doctors as well as parents through the IoT cloud system and also ambulance in case of any emergency. The collected data is then sent to doctors as well as parents through the IoT cloud system and also ambulance in case of any emergency.

\section{Related Work}

There many papers based on health monitoring of adult health care. The paper proposes to monitor the health parameters such as temperature, pulse, and respiration and giving them an appropriate alert and feedback using Bluetooth protocol. The proposes giving care to older adults by monitoring their sleeping conditions using a mattress sensor and giving them suggestions and advice to lead a good sleep. The [1] proposes to remotely monitor the person with cardiovascular disease and help them to improve their health by giving physician advice. The [2-8] proposes Respiration rate and heart rate can be monitored visually using an IR sensor and capture video and implement it in an image processing algorithm. The [9] proposes a Wearable shoe-based sensor to monitor posture allocation and activities to estimate energy expenditure that may aid in obesity prevention and treatment. The [10] wireless patient monitoring using sensors and pulse-oximetry based surveillance system advanced display and information system. The [6] proposes a remote 
monitoring system for a patient with cardiovascular disease using an android smart phone application using Bluetooth and cellular networks. The [3] proposes patient monitoring in hospitals using pulse oximeters and mobile ECG and giving the alarm. The [7] wearable sensor for monitoring using biosensors and transmitted the data to medical centers. The paper [8] proposes A wireless body sensor network for arterial pulse wave (PW) measurements is presented $[11,12]$. The paper [5] proposes A wireless wearable multi senor integrated measurement system (WIMS) for measurement of energy expenditure and breathing volume monitoring. The [13] proposes the in-vehicle nonintrusive biopotential measurement system for driver health monitoring and fatigue detection.

\section{Design Methodology}

This system is going to monitor the health condition of the patients to identify the basic functions of the body that will result in whether he/she is normal or in an abnormal condition. The developed system that can monitor the vital signs of the patient using various sensors parameters and the sensed values can be transmitted to the microcontroller. Then the microcontroller transmits the data to the cloud server through the dashboard losant and displays it in MQTT mobile application (see fig 1). So, this leads to continuous monitoring and it will help the patient's health and also immediate treatment will be given when an abnormality detected. Then the database will be collected from losant and implemented in the machine learning algorithm. The proposed system monitors the health condition by measuring various sensor parameters. The Breath sensor, Heartbeat sensor, temperature sensor, MEMS sensors are used to monitor the health status of the infant. The motion sensor senses the external human motion. The sensors are interfaced with the microcontroller and the controller is powered from 5-12V. After powering up the controller, the controller starts sensing the sensor parameters. There is a threshold point set for each of the sensor parameters.

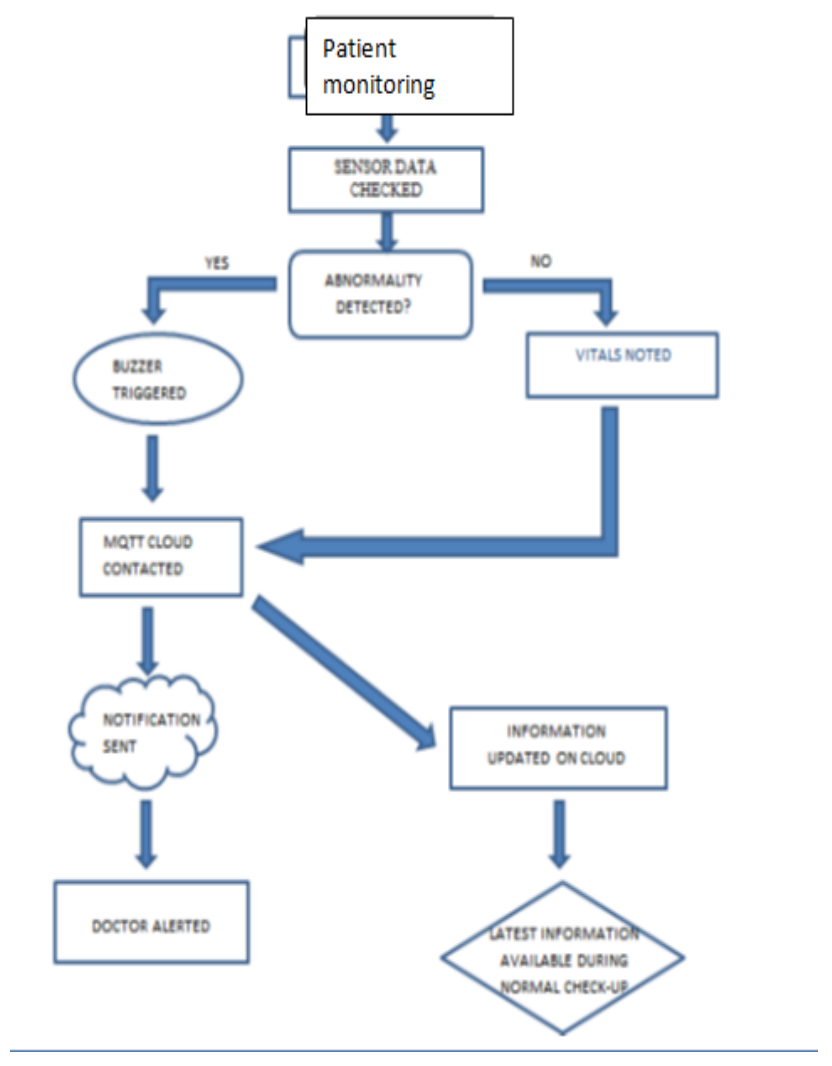

Fig 1. Flow chart 
If the sensor parameters reach beyond the threshold point, the parameter is abnormal. The controller is connected with the Wi-Fi and the cloud server. If the Wi-Fi is enabled, the controller automatically sends the sensed parameters to the cloud server and displays the status of the patient through a mobile application and sends an SMS and Gmail to the doctors and caretakers.

\section{System Architecture}

The architecture of the patient's health monitoring system consists of both hardware and software. The block diagram is as shown in Fig. (2) And consists of the modules discussed in detail below.

The architecture of our proposed system consists of four major parts.

1. Sensor nodes- sensors for collecting the vital signals from the patient.

2. A computing system, which processes the signals into interpretable data.

3. Display and alert unit-to display readings on a PC/mobile or to alert the concerned authority in case of any abnormality using buzzers.

4. Cloud computing or wireless transmission and presenting the received-over the air for remote monitoring and notification.

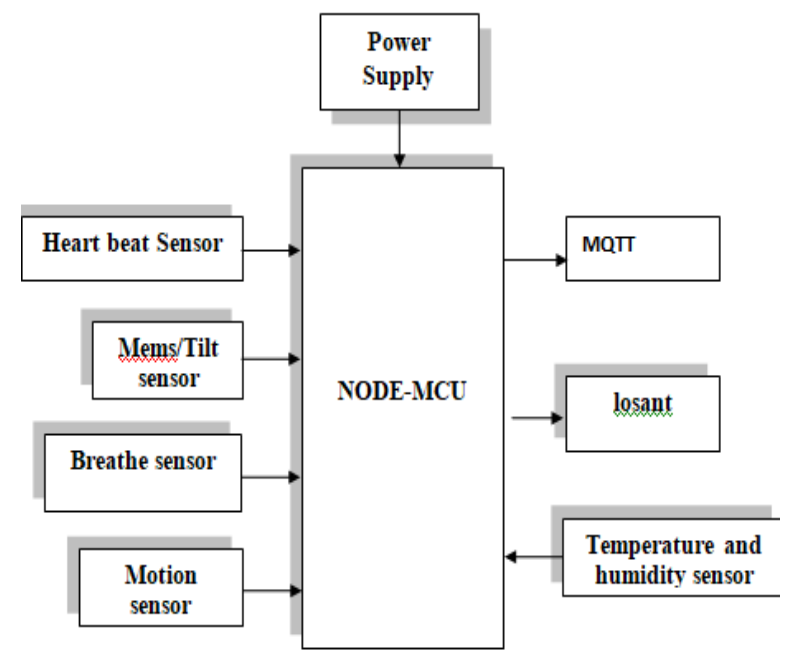

Fig 2. Block Diagram

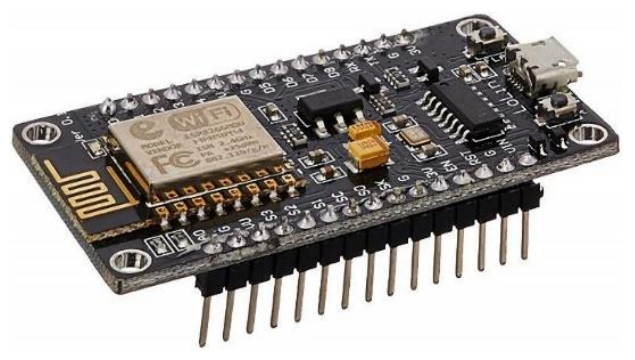

Fig 3. Node MCU

\section{Hardware Implementation}

\section{A. NodeMCU}

NodeMCU is a microcontroller platform that has inbuilt Wi-Fi in it (see fig 3). Because of the inbuilt Wi-Fi NodeMCU is preferred in the Internet of Things (IoT) based application. NodeMCU 
is most integral hardware structure, which has 11 digital pins, 1 analog pin, 1 data port and other pins for communication Node MCU controller collectors all the sensor data and transmitted it to the cloud server and MQTT mobile application through wifi.

\section{B. Breath sensor}

The breath sensor is used to measure the breath and suffocation status of the patient by using MO26 sensor. It has a microphone that detects the wheezing sound of the patient and detects whether the breath is normal and abnormal according to the threshold point set for the sensor.

\section{DHT 11}

This DHT11 Temperature and Humidity Sensor features a calibrated digital signal output with the temperature and humidity sensor capability. The DHT11 temperature and humidity sensor measure the temperature and humidity parameter of the patient. If the temperature exceeds beyond the threshold then it is abnormal.

\section{Pulse sensor}

Heartbeat Sensor is an electronic device that is used to measure the heart rate i.e. speed of the heartbeat. the heart rate can be measured by the sensor having a light emitting LED and a light detector. The light absorbed by the finger tissue and reflects it which is detected by light detector for 15 seconds and counts the vibration of the heart beat through finger tissues. If the beat exceeds the threshold then it is abnormal.

\section{E. PIR Sensor}

A PIR sensor is an electronic sensor that measures infrared light radiating from objects in its fields of view. PIR sensors detect general movement. Here the PIR sensor detects the external human motion inside the room. if the digital pin is high, then motion detected, otherwise no motion is detected.

\section{F. Tilt Sensor}

The tilt sensor is used to monitor the sleeping position of the patient to check whether the patient is lying on the bed or fell. If the angle exceeds the threshold, then it indicates felt down.

\section{Message Queuing Telemetry Transport (MQTT) protocol}

MQTT is a lightweight telemetry protocol used for communication. It is widely used in machine-to-machine (M2M) communication. A basic operation in the MQTT protocol is Publish and Subscribe to a particular to a given topic. This information about the topic sent to a server by a broker. The broker pushes data to a client which is subscribed to a particular client topic. It is widely used in the resource-constrained IoT devices. It is a mobile application that displays the collected sensor data through it through Wi-Fi.

\section{Data processing visualization using Losant}

The "dashboard" is often displayed on a web page which is linked to a database that allows the report to be constantly updated. Using the losant we integrate the data and the collected sensor data will be sent to firebase. From the firebase, we fetch the data for processing the live algorithms and real-time android application through which one can analyze the real-time constraints faced by the patient also we can predict the future threat possibilities that can cause for the patient. 


\section{Machine learning algorithm application}

Machine learning is an artificial intelligence-based application that provides the ability of the system to learn and process automatically without the explicit program. Here it mainly focuses on data access, data analysis, false data removal, and the prediction.

\section{Random Forest Algorithm}

Random Forest could be a versatile, straight forward to use machine learning algorithmic rule that produces, an excellent result most of the time. It is additionally one in every of the foremost used algorithms, as a result of its simplicity and also the undeniable fact that it is often used for each classification and regression tasks (see fig 4 and fig 5). Random Forest is a supervised learning algorithm. Like it is already noticed clearly from its name, it creates a forest and makes it somehow random. The "forest" it builds, is an ensemble of Decision Trees, most of the time trained with the "bagging" method. The random forest algorithm is used in a lot of different fields, here the collected data from sensors is applied to this algorithm. it is used to identify the exact combination of the data through which you can predict future data and also, we can eliminate the false data.
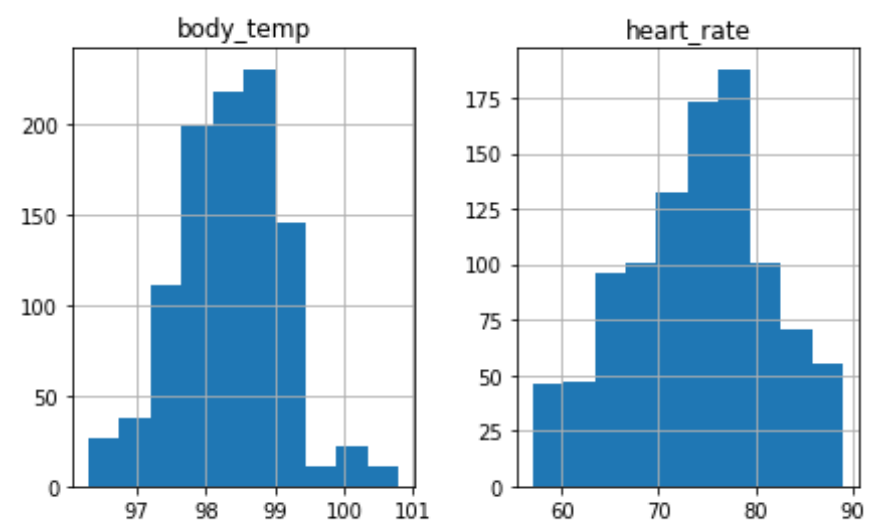

Fig 4. Graphical representation from random forest

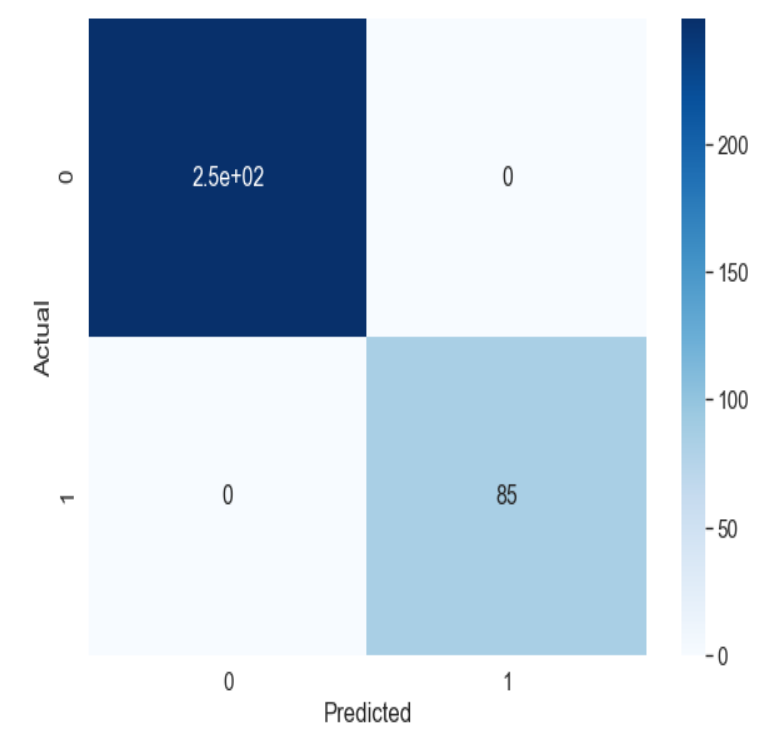

Fig 5. Actual and predicted data using random forest 


\section{Conclusion}

IoT based cloud agent system for patient's health monitoring system using wireless sensor networks and machine learning is proposed. Through this system, the patient's health condition is continuously monitored and giving them better care.

\section{References}

[1] N. Alshurafa, J. A. Eastwood, S. N. Jason J. Liu, Wenyao Xu, Hassan Ghasemzadeh, Mohammad Pourhomayoun, Majid Sarrafzadeh, Improving Compliance in Remote Healthcare Systems Through Smartphone Battery Optimization., IEEE Journal of Biomedical and Health Informatics, 19 (2014) 57-63.

[2] K. Zhu, M. Li, S. Akbarian, M. Hafezi, A. Yadollahi, B. Taati, Vision-Based Heart and Respiratory Rate Monitoring During Sleep - A Validation Study for the Population at Risk of Sleep Apnea, IEEE Journal of Translational Engineering in Health and Medicine, 7 (2019) $1-8$.

[3] L. Clifton, D. A. Clifton, M. A. Pimentel, P. J. Watkinson, L. Tarassenko, Predictive Monitoring of Mobile Patients by Combining Clinical Observations with Data from Wearable Sensors, IEEE Journal of Biomedical and Health Informatics, 18 (2013) 722-730.

[4] M. H, Li, A. Yadollahi, B. Taati, Non-Contact Vision-Based Cardiopulmonary Monitoring in Different Sleeping Positions, IEEE Journal of Biomedical and Health Informatics, 21 (2016) 1367-1375.

[5] L. Mo, S. Liu, R. X. Gao, D. John, J. W. Staudenmayer, P. S. Freedson, Wireless Design of a Multisensor System for Physical Activity Monitoring, IEEE transactions on Biomedical Engineering, 59 (2012) 3230-3237.

[6] N. Alshurafa, C.Sideris, M. Pourhomayoun, H. Kalantarian, M. Sarrafzadeh, J. A. Eastwood, Remote Health Monitoring Outcome Success Prediction using Baseline and First Month Intervention Data, IEEE Journal of Biomedical and Health Informatics, 21 (2016) 507-514.

[7] A. Pantelopoulos, N. G. Bourbakis, A Survey on Wearable Sensor-Based Systems for Health Monitoring and Prognosis, IEEE Transactions on Systems, Man, and Cybernetics, Part C (Applications and Reviews), 40 (2009) 1-12.

[8] M. Peltokangas, A. Vehkaoja, J. Verho, M. Huotari, J. Röning, J. Lekkala, Monitoring Arterial Pulse Waves with Synchronous Body Sensor Network, IEEE Journal of Biomedical and Health Informatics, 18 (2014) 1781-1787.

[9] E. S. Sazonov, G. Fulk, J. Hill, Y. Schutz, R. Browning, Monitoring of Posture Allocations and Activities by A Shoe-Based Wearable Sensor, IEEE Transactions on Biomedical Engineering, 58 (2010) 983-990.

[10] S. P. McGrath, I. M. Perreard, M. D. Garland, K. A. Converse, T. A. Mackenzie, Improving Patient Safety and Clinician Workflow in The General Care Setting with Enhanced Surveillance Monitoring, IEEE journal of biomedical and health informatics, 23 (2018) 857866.

[11] M. Takahara, K. Nakamura, F. Huang, I. Tanev, K. Shimohara, (2018) Caregiver Support System for Nursing Care for Older Adults, In 2018 5th Asia-Pacific World Congress on Computer Science and Engineering (APWC on CSE), IEEE, 121-124.

[12] A. K. Triantafyllidis, V. G. Koutkias, I. Chouvarda, N. Maglaveras, (2012) A Pervasive Health System Integrating Patient Monitoring, Status Logging, and Social Sharing, IEEE journal of biomedical and health informatics, 17 (2012) 30-37.

[13] Y. Sun, X. Yu, An Innovative Nonintrusive Driver Assistance System for Vital Signal Monitoring, IEEE Journal of Biomedical and Health Informatics, 18 (2014) 1932-1939. 


\section{Acknowledgement Nil}

\section{Funding}

This study was not funded by any grant

\section{Conflict of interest}

None of the authors have any conflicts of interest to declare.

\section{About The License}

The text of this article is licensed under a Creative Commons Attribution 4.0 International License

\section{Cite this Article}

V. Prasanna kumari, C. Amutha, IoT Based Cloud Agent System for Adult Health Care Monitoring, International Research Journal of Multidisciplinary Technovation, Vol 2, Iss 5 (2020) 35-41.

DOI: https://doi.org/10.34256/irimt2056 